\title{
Can gravitational waves predispose neuro-cardiovascular circadian rhythms?
}

\author{
Singh RB ${ }^{1 *}$, Sergey Chibisov ${ }^{2}$, Sergey Shastun ${ }^{2}$, Rie Horiuchi $^{3}$ and Toru Takahashi ${ }^{4}$ \\ ${ }^{1}$ Halberg Hospital and Research Institute, Moradabad, Uttar Pradesh, India \\ ${ }^{2}$ People's Friendship University of Russia, Moscow, Russia \\ ${ }^{3}$ Department of Food science and Nutrition, Faculty of human Environmental Sciences, Mukagawa Women's University, Japan \\ ${ }^{4}$ Graduate School of human Environment Medicine, Fukuoka, Women,s University, Fukuoka, Japan
}

Environmental factors such as geomagnetic forces, Western diet, physical inactivity, tobacco and alcoholism are primary risk factors for physical, social, spiritual and mental diseases of the individuals and populations [1]. Late night eating and late night sleep as well as sleep deprivation due to any cause can predispose circadian disruption of cardiovascular rhythms resulting in increased risk of cardiovascular diseases and diabetes [1-3]. These risk factors and risky behaviors are not given much consideration, which could be important confounders during clinical trials with drugs. Majority of the trials try to compare two agents; intervention and placebo showing that one therapy is superior to another without much consideration of these risk factors, hence final results are open to bias. The beneficial effects attributed to therapy in the intervention group could be due to these confounders. A poor mental and spiritual health can promote neuropsychiatric dysfunctions characterized with wars and terrorism among social groups, politicians and public as well as cardiovascular diseases; hypertension, sudden cardiac death, acute coronary syndromes and strokes [3-7]. It is possible that these socio-political problems may be prevented by treating chronobiological risk factors; late night sleep, late night eating, heavy meals, variability in biological functions according to 24-hour cycle. Physical activity, diet characterized with a low w-6/w-3 ratio Mediterranean style diet may also be a confounder in many trials. This view point aims to highlight these confounders for identification during planning of the clinical trials and to emphasize that gravitational waves can influence neuro-cardiac function which could be unknown confounders.

Space weather hazards have been well-studied during the past few decades, mainly in connection with effects on technical systems [2-7]. However, the biological effects of solar and geomagnetic activity have been largely ignored because the amplitudes of the electro- magnetic fields (EMF) are small, typically one to several hundreds of nano-teslas $[2,3]$. This is much weaker than electromagnetic noise of anthropogenic origin and about ten orders of magnitude less than the characteristic energies of biochemical reactions. It is proposed that amplitudes of electromagnetic fields may be similar to that of the amplitudes of the gravitational waves. Recently, Breus, et al. reported the effect of geomagnetic activity on the regulation of heart rhythm of cosmonauts during the expeditions on board of the Soyuz spacecraft, and the MIR orbital space stations showing adverse effects on heart rhythm depending on the duration of the flight and preexisting integrity of the myocardium [2-4]. Laser Interfero- meter Gravitational-Wave Observatory (LIGO) has announced the discovery of gravitational waves on the earth from the cosmos in Sept 2015, which were predicted by Albert Einstein, about 100 years ago, may also be the cause of concern for cardiovascular dysfunction among cosmonauts. (Figure 1).

All the cosmonauts were examined under flight conditions outside the geomagnetic disturbances and in ground preflight conditions, during disturbances and without disturbances [2-4]. The four series of analytical researches in which the data about changes of characteristics of heart rate variability are demonstrated for the first time in the presence of specific effect of geomagnetic disturbances on the system of vegetative regulation of blood circulation of cosmonauts during the flight. The response of cosmonauts' heart rhythm on the magnetic storm is depending on the initial functional background and, in particular on the state of mechanisms of vegetative regulation; the duration of flight and endogenous capability of adaptation to environment [2].

The effects of gravitational waves on space and whether hazards are not known. Gravitational waves are reported as the elusive ripples in the fabric of space-time in September 2015 which were detected by the twin detectors as a tiny vibration passing through our planet. The main source of the waves were two inward spiraling black holes that smashed together to form a single black hole. It is a highly cataclysmic, space-warping event for all the scientists of the world. Because, findings will open up an entirely new era of scientific investigation; unknowable details of some of the universe's most violent events; from neutron star and binary black hole mergers, to supernova explosions. Further

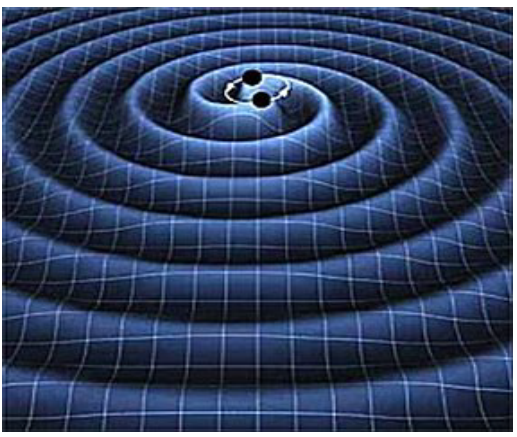

Figure 1. Gravitational waves produced by orbiting of two black holes.

Correspondence to: Singh RB, Halberg Hospital and Research Institute, Moradabad, Uttar Pradesh, India, Tel: +91-5912417437; E-mail: rbs@tsimtsoum.net

Received: October 21, 2016; Accepted: November 11, 2016; Published: November 14, 2016 
research may quantify the effects of magnetic forces in relation to gravitational waves of the cosmos on earth and on biological function and dysfunction.

Recent evidence indicate that geomagnetic forces, biological and physical cycles, solar activity and magnetic storms can predispose non-communicable diseases; CVDs; myocardial infarction, stroke, sudden cardiac death, aggression, depression and hyperactivity anxiety disorders (3-7). Apart from these environmental factors; diet, physical inactivity, alcoholism, lack of mental and spiritual health can also predispose many of the NCDs; hypertension, heart attack, type 2 diabetes, aggression, anxiety, depression and brain dysfunctions [511]. Space weather continues to influence human physiopathology, biological and metabolic functions which are coordinated by the supra-chiasmatic nuclei, widely (but not unanimously) viewed as the central circadian oscillator [12]. Peripheral clock functions are also important, and when disturbed, may lead to metabolic dysfunction in liver and beta cells of pancreas, predisposing hyperglycemia, blood glucose variability and blood pressure variability [12]. Cornelissen, et al. has reported on circadian disruption of the blood pressure rhythm as predictor of adverse cardiovascular outcome and overall mortality which may be regulated by circadian clocks [13] (Figure 2).

The terrorist attacks in USA, UK, Spain and India in the last 15 years and the responses of the politicians and military action could be a manifestation of poor status of mental and spritual health of the decision makers. A better human security measures, taken by these nations could have been an excellent symptomatic treatment for prevention of these attacks. However, such measures would not be able to eradicate terrorism and wars that have occurred in the last several thousand years in the past and are likely to occur in the near future. It may be proposed that individuals who commit acts of violence are 'statistically more likely to have a background of poor mental health' than the rest of the population. They may be also exposed to the effects of the solar activity and magnetic forces or gravitational waves of the cosmos on body functions resulting in to neuropsychiatric dysfunction. However, poor mental health on its own does not lead directly to wars and terrorism and may need a social and behavioral stimulus. However, it is difficult to say that mental ill-health is not a factor here. It is difficult to imagine that the perpetrator of a frenzied knife attack on a 60-year-old woman, as happened in Russell Square, this year in 2016, was in good mental health. It seems that mental health is not the sole or determining factor in these cases and in some disturbances in cosmos may be predisposing factors. There may be social, cultural

\section{Entrainment of circadian clock}

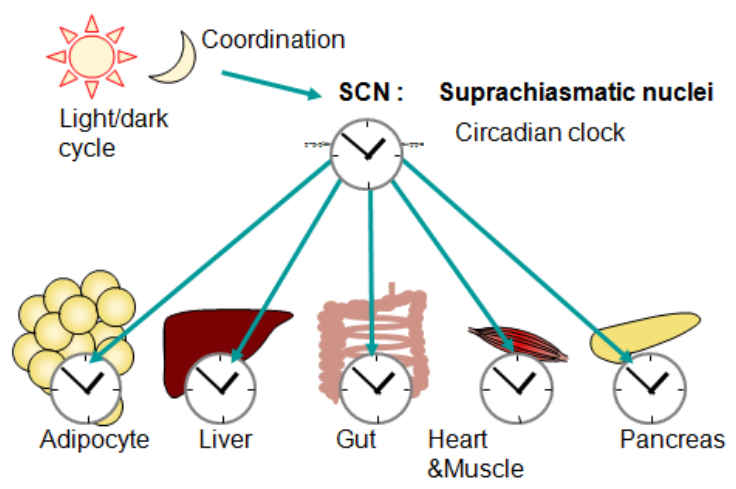

Figure 2. Effects of circadian clock disruption possibly by gravitational waves/geomagnetic activity on earth, on biological rhythms in various organs. and economic factors to be considered, not to mention personal factors involving family, friends and community. It is important to study, how an individual responds to disappointment and rejection, for instance, is mediated by his or her sense of belonging and purpose. It is possible that the causes of cardiovascular diseases(CVDs) and neuropsychiatric dysfunctions as well as terrorism and wars may have a unifying hypothesis, which lies in the environment, that is known to have possible influence on physical, social, mental and spiritual health of the individuals and populations [1-8]. Halberg, et al. proposed that our body is exposed to cosmos and works as magnometer and cosmometer by measuring geomagnetic forces [8].

In brief, it may be proposed that gravitational waves can also be measured as a disturbance of biological function via circadian clock and or peripheral clocks, in our body systems as chronobiological dysfunction. It is possible that gravitational waves may have similarity with geomagnetic forces present in the cosmos or biosphere and may be associated with occurrence of CVDs, and neuropsychiatric dysfunctions, resulting in to wars and terrorism [9-11]. These risk factors should be duly considered during planning of the clinical trials.

\section{References}

1. Zadeh SS, Pahlevanlo A, Rad NG, Jalili M, Singh RB, et al. (2011) Can diet and lifestyle factors and geomagnetic forces predispose aggression. The Open Nutr J 4: 176-179.

2. Breus TK, Baevskii RM, Chernikova AG (2012) Effects of geomagnetic disturbances on humans functional state in space flight. JBiSE 5: 341-355.

3. Breus TK, Halberg G, Cornelissen G (1995) Effect of solar activity on the physiologica rhythms of biological systems. Biofizika 40: 737-748. [Crossref]

4. Breus TK, Cornelissen G, Halberg F, Levitin AE (1995) Temporal associations of life with solar and geophysical activity. Annales Geophysicae 13: 1211- 1222.

5. Syutkina EV, Cornelissen G, Grigoriev AE, Mitish MD, Turti T, et al. (1997) Neonatal intensive care may consider associations of cardiovascular rhythms with local magnetic disturbance. Scripta Medica 70: 217-226.

6. Chibisov SM, Breus TK, Levitin AE, Drogova GM (1995) Biological effects of a global magnetic storm. Biofizika 40: 959-968.

7. Cornelissen G, Halberg F, Breus TK, Syutkina EV, Baevskii RM, et al. (2002) Nonphotic solar associations of heart rate variability and myocardial infarction. Journal of Atmospheric and Solar-Terrestrial Physics 64: 707-728.

8. Halberg F, Cornélissen G, Regal P (2004) Chronoastrobiology: Proposal, nine conferences, heliogeomagnetics, transyears, near-weeks, near-decades, phylogenetic and ontogenetic memories. Biomed Pharmacother 58: 150-187.

9. Singh RB, Fedacko J, Sharma JP, Vargova V, Sharma R, et al. (2010) Association of inflammation, heavy meals, magnesium, nitrite, and coenzyme Q10 deficiency and circadian rhythms with risk of acute coronary syndromes. World Heart Journal 2: 219228.

10. Hammoudeh AJ, Alhaddad IA (2009) Triggers and the onset of acute myocardial infarction. Cardiol Rev 17: 270-274. [Crossref]

11. Singh RB, Pella D, Neki NS, Chandel JP, Rastogi S, et al. (2004) Mechanisms of acute myocardial infarction study (MAMIS). Biomed Pharmacother 58 Suppl 1: S111-115. [Crossref]

12. Singh RB, Shastun S, Hristova K, Fedacko J, Joshi P, et al. (2016) Blood pressure and blood glucose variability, the silent killers, in subjects with diabetes mellitus, flying blue. World Heart J 8: 109-120.

13. Cornelissen G, Siegelova J, Otsuka K (2016) Circadian disruption of the blood pressure rhythm as predictor of adverse cardiovascular outcome and overall mortality. World Heart J 8: 5-10.

Copyright: (C2016 Singh RB. This is an open-access article distributed under the terms of the Creative Commons Attribution License, which permits unrestricted use, distribution, and reproduction in any medium, provided the original author and source are credited. 